\title{
El derecho a la educación superior en tiempos de pandemia en un Estado constitucional de derechos.
}

The right to higher education in pandemic times inside a Constitucional State of Laws

\author{
Farid Villacís De La Cueva* \\ Investigador jurídico independiente \\ José Paredes Sandoval** \\ Investigador jurídico independiente
}

Infomación del Artículo

Original - Ruptura, 2020

Artículo recibido / Received: 09 de octubre, 2020

Artículo aceptado / Accepted: 22 de noviembre, 2020

\section{Citación}

Villacís, F. y Paredes, J. (2020). El derecho a la educación superior en tiempos de pandemia en un Estado constitucional de derechos. Revista Ruptura de la Asociación Escuela de Derecho PUCE. Edición 2020, p (49-87).

DOI: $10.26807 /$ rr.vi02.34

Resumen: El derecho a la educación forma parte del núcleo duro de los Derechos Económicos, Sociales y Culturales (DESC), ya que es un medio intrínseco e indispensable para alcanzar una vida digna. El Estado tiene la obligación de garantizar este derecho, permitir el

* Presidente de la Asociación Escuela de Derecho de la Pontificia Universidad Católica del Ecuador. Egresado de la Facultad de Jurisprudencia de la PUCE. Correo electrónico: faridv.08@hotmail.com

** Defensor Estudiantil de la Asociación Escuela de Derecho de la Pontificia Universidad Católica del Ecuador. Egresado de la Facultad de Jurisprudencia de la PUCE. Correo electrónico: josed.paredes98@gmail.com 
acceso sin discriminación y la continuidad del estudiante en el sistema educativo, el cual debe adaptarse a las condiciones sociales y culturales. En una circunstancia compleja como es una pandemia a causa del COVID-19, las actuaciones estatales y decisiones jurisprudenciales de la Corte Constitucional del Ecuador, trazan el camino y marcan precedentes para el goce efectivo de este derecho, pues la educación superior posibilita el desarrollo de un país y su sociedad. En tal virtud, el Estado debe respaldar este derecho por medio de sus actuaciones estatales: regulación normativa amparada en garantizar derechos y respetar principios; políticas públicas que tracen actuaciones en estricto apego al ordenamiento jurídico y, en el supuesto que no ocurra, la Corte Constitucional será el órgano jurisdiccional encargado de velar por la garantía y respeto a la educación superior.

Palabras claves: Derecho a la educación, educación superior, autonomía universitaria, cogobierno, Corte Constitucional, acción de incumplimiento, acción de inconstitucionalidad.

Abstract: The right to education is part of the nucleus of the Economic, Social and Cultural Rights due to its intrinsic and indispensable effect in achieving a dignified life. This right must be guaranteed by the Government, who has the duty to allow its access without discrimination and ensure the student's continuity in the educational system which has to adapt to the social and cultural conditions. In a complex circumstance such as a pandemic caused by COVID-19, state actions and jurisprudential decisions of the Constitutional Court of Ecuador trace the path for the effective enjoyment of the rigth to education, hence this right enables the development of a country and its society. In addittion to this Government must support the right to education through the following actions: create a normative regulation based on guaranteeing rights and respecting principles, and establish public policies in strict adherence to the legal system. Otherwise, the Constitutional Court of Ecuador will be the competent authority in charge of protecting and guaranteeing the rigth to education.

Keywords: Right to education, higher education, university autonomy, co government, Constitutional Court, action of unconstitutionality. 


\section{Introducción}

La educación es la exploración de un sinnúmero de ciencias y actividades que va desarrollando e investigando el ser humano a lo largo de su vida, pues, al ser un ente racional, le ha permitido evolucionar y mantenerse en un constante descubrimiento, fortaleciendo las capacidades y raciocinio. La educación forja hombres y mujeres libres, pues permite el desarrollo continuo y reduce el desconocimiento y la incertidumbre al dotar a la persona de ideas, conocimientos y habilidades para potenciar su individualidad.

La educación va más allá de proporcionar un aprendizaje referente a distintos saberes, debido a que es el medio de vital importancia para el desarrollo de la persona y de la sociedad. La importancia de la misma se refuerza al ser reconocida como un derecho humano e impone un deber al Estado de garantizar el ejercicio de dicho derecho. En este sentido, la educación ecuatoriana se desarrolla a través de varios niveles, los cuales son: educación inicial, educación básica, bachillerato y educación superior ${ }^{1}$.

Con respecto a las niñas, niños y adolescentes, el derecho a la educación tiene una importancia fundamental, pero el mismo no se agota en ellos, sino que continúa hasta la adultez y la persona se puede educar en cualquier etapa de su vida. En este contexto, cobra sentido la noción de educación fundamental entendida como aquella que satisface las necesidades básicas de aprendizaje e inspira la educación de personas adultas (CLADEM, 2011, p.9-10).

La educación de tercer nivel se compone de varias instituciones, las cuales son las universidades, las escuelas politécnicas, los institutos superiores técnicos o tecnológicos, pedagógicos, instituciones de arte y conservatorios superiores, ya sean de carácter públicas o privadas (LOES, 2010, art.14), las cuales mantendrán la fuerte garantía constitucional de la autonomía universitaria, punto central que se aborda en la presente investigación.

1 La presente investigación se referirá únicamente a la educación superior. 


\section{Breves antecedentes de la educación superior en el Ecuador}

La República del Ecuador, en 1830, reconoció la importancia de la educación, en especial de la pública, ya que en la normativa constitucional vigente de la época se prescribió que la educación debía ser promocionada por la Función Legislativa, a través del Congreso Nacional (CE, 1830, art.26, num.7). Desde inicios de la vida republicana, el Estado adoptó en su normativa un compromiso para respetar y garantizar el acceso a la educación, empero la educación universitaria era concebida como un privilegio en aquella época.

Si bien el reconocimiento normativo en el Ecuador de la educación en general inicia desde la vida republicana, no se debe dejar de señalar la importancia de la educación superior en la región Latinoamericana, que se remonta a principios del siglo XVI, cuando se fundó la Universidad de Santo Domingo y, posteriormente, la Universidad Pontificia de San Marcos en Lima y la Real y Pontificia Universidad de México (Ferreyra, Avitabile, Botero, Haimovich y Urzúa, 2017, p.2).

En la Real Audiencia de Quito, espacio territorial donde se encuentra el actual Ecuador, la historia de la universidad data de 1586, cuando los frailes agustinos fundaron la Universidad San Fulgencio (Pareja, 1986). En septiembre de 1620 se fundó la Universidad San Gregorio Magno, universidad génesis de la que es actualmente la Universidad Central del Ecuador.

De los acontecimientos históricos relevantes se considera que, en 1906, el Ecuador expide la Ley Orgánica de la Instrucción Pública, la cual determinó que la instrucción pública sería prioritaria en los establecimientos nacionales sostenidos por el Estado y la enseñanza superior comprendía en ese tiempo siete facultades, entre ellas la de Jurisprudencia (OEI, s.f). Desde el enfoque Latinoamericano, Argentina llevó a cabo un hecho histórico, revolucionario y considerado un precedente fundamental para la educación superior: la Reforma de Córdoba de 1918, la que propugnó la gratuidad como el medio de acceso y la lucha por la democratización. 
La Reforma de Córdoba tiene su origen en las demandas crecientes de la población migrante europea en Argentina, personas que trajeron ideas novedosas relacionadas con la vida de la clase media y el sector obrero y que reclamaban la necesidad de la educación superior para la democratización de la sociedad (Andrade Ortega, 2017). En la Argentina de la época, la educación superior era controlada exclusivamente por el clero y los terratenientes, lo cual coartaba el acceso a la educación de las demás clases de la sociedad; ante esta situación, en la Universidad de Córdoba se implementó una reforma que favoreció la autonomía universitaria y el cogobierno, ampliación de la cobertura educativa a distintas clases sociales y la expansión de un modelo gratuito y monopólico con participación del Estado (Cóndor, 2018).

Olivier (2012), al analizar las circunstancias y condiciones de la población latinoamericana, en relación a la posibilidad de ingreso a la educación superior, señala que "el paso de una universidad reservada a los grupos privilegiados a un sistema de apertura más democrática es uno de los saltos más relevantes en el siglo XX" (p.2).

Años posteriores, en las diversas Constituciones ecuatorianas y a partir de la segunda mitad del siglo XX, el constituyente estableció como un deber primordial del Estado el garantizar el acceso a la educación en general (OEI, s.f). En la Constitución ecuatoriana de 1979, el Estado previó la gratuidad de la educación en todos los niveles, incluido el universitario (Simon, 2005). En la Constitución antedicha se prescribió que el Estado tenía la obligación de destinar, al menos, el 30\% de los ingresos corrientes al sector educativo (CE, 1979, art. 71). La normativa constitucional referente a la educación superior en 1979 no sufrió mayores modificaciones en la Constitución de 1998, pero en la norma constitucional de 2008 se reconoce que la educación superior no puede limitarse en razón de ninguna discriminación que conculque el ejercicio de los derechos de las personas.

En la década de los noventa puede observarse el mayor crecimiento de instituciones superiores en la historia educativa de la región latinoamericana (Olivier, 2012, p.1), esto permitió una mayor cantidad de personas ingresar a la educación de tercer nivel. La educación superior, de manera globalizada, se ha expandido considerablemente durante el 
último siglo, donde las personas en edad universitaria han pasado de representar cerca del 1\% en el año 1900, hasta alcanzar alrededor del $20 \%$ de esa cohorte para el año 2000, cifra que no se aleja de la realidad ecuatoriana (Rivera, 2019, cita a Schofer \& Meyer, 2005).

\section{El derecho a la educación superior desde un enfoque global}

En el ordenamiento jurídico ecuatoriano vigente se concibe a la educación como un deber ineludible del Estado y un derecho que tendrán las personas para ejercerlo (CRE, 2008, art.26), el cual incluye la permanencia y el acceso a las instituciones educativas, aplicables a toda institución y proceso educativo (CC, Sentencia 189410-JP/20, 2020).

Conforme lo dicho anteriormente, en 2008 el constituyente establece el deber prioritario del Estado con respecto a la educación superior; se la concibió como un derecho de las personas a lo largo de su vida, por lo cual las políticas públicas y la inversión estatal propenderá al acceso, permanencia y egreso sin limitación alguna (CRE, 2008, arts. 26, 27 y 28).

A parte de lo mencionado, la norma constitucional prevé que la educación tendrá un enfoque antropocéntrico, ya que el ser humano es el centro del derecho a la educación (CRE, 2008, art.27); reconoce que la educación pública mantendrá un principio universal y será laica en todos sus niveles; y será gratuita hasta la educación superior (CRE, 2008, art.28). Textualmente, la Constitución establece que:

Artículo. 356.- La educación superior pública será gratuita hasta el tercer nivel.

El ingreso a las instituciones públicas de educación superior se regulará a través de un sistema de nivelación y admisión, definido en la ley. La gratuidad se vinculará a la responsabilidad académica de las estudiantes y los estudiantes. 
Con independencia de su carácter público o particular, se garantiza la igualdad de oportunidades en el acceso, en la permanencia, y en la movilidad y en el egreso, con excepción del cobro de aranceles en la educación particular.

El cobro de aranceles en la educación superior particular contará con mecanismos tales como becas, créditos, cuotas de ingreso u otros que permitan la integración y equidad social en sus múltiples dimensiones.

La norma constitucional (CRE), vista de una manera integral y sistémica como la conciben Navas y Chiliquinga (2019), en relación a la educación superior plantean dos aristas:

En primer lugar, se trata de un derecho subjetivo, que denominaremos derecho a la educación superior, cuya base es el más amplio derecho a la educación. Además de este derecho subjetivo de alcance general, se desprenden varios derechos específicos, como son el derecho de acceder a una educación gratuita hasta el tercer nivel. En segundo lugar, la educación superior se concibe como una actividad sin fines de lucro, desarrollada a través de un sistema y una serie de mecanismos que la garantizan. Esta manera de constitucionalizar la educación superior permite combinar la protección propia del derecho subjetivo con una protección objetiva a través de una institucionalidad garantista (p.199).

La educación se entiende como un derecho subjetivo y también denota un sentido dentro y fuera de las instituciones educativas. El derecho a la educación como derecho subjetivo consiste en la facultad o prerrogativa de la persona para alcanzar una habilidad, conocimiento o alguna enseñanza útil que dé al titular del derecho una ventaja individual (Cotino Hueso, 2012). Por otro lado, el derecho a la educación desde dentro y fuera de las instituciones educativas consiste en la formación en valores, cultura, religión, deporte o cualquier enseñanza, personal o profesional, a lo largo de la vida de la persona (Cotino Hueso, 2012).

La educación es un derecho constitucionalizado que permite el ejercicio de los derechos económicos, sociales y culturales (DESC), pues la educación garantiza un desarrollo de la persona y la sociedad; 
la misma se encuentra tutelada a través de un sistema y mecanismos que posibilitan de este derecho una realidad. Ahora bien, la educación mantiene un rango constitucional e internacional, ha sido reconocida como un derecho interdependiente y de carácter imperante a ser garantizado por parte de los Estados.

El Pacto Internacional de Derechos Económicos, Sociales y Culturales (PIDESC), instrumento internacional de obligatoria observación y cumplimiento, ratificado por el Ecuador en 1969 y con su entrada en vigor en 1976, dispone:

Artículo 13.- 1. Los Estados Partes en el presente Pacto reconocen el derecho de toda persona a la educación. Convienen en que la educación debe orientarse hacia el pleno desarrollo de la personalidad humana y del sentido de su dignidad, y debe fortalecer el respeto por los derechos humanos y las libertades fundamentales. Convienen asimismo en que la educación debe capacitar a todas las personas para participar efectivamente en una sociedad libre, favorecer la comprensión, la tolerancia y la amistad entre todas las naciones [...]

c) La enseñanza superior debe hacerse igualmente accesible a todos, sobre la base de la capacidad de cada uno, por cuantos medios sean apropiados, y en particular por la implantación progresiva de la enseñanza gratuita. (énfasis añadido).

La educación superior radica en comprender que ésta es un bien público de carácter social, un derecho humano del cual deriva su universalidad e impone la obligación al Estado de garantizar a las personas su acceso, permanencia y egreso para generar sociedades más desarrolladas, equitativas y productivas (Rivera, 2019).

La III Conferencia Regional de Educación Superior para América Latina y el Caribe (CRES) contempló a la educación superior como un bien público, social-estratégico, un derecho humano y universal y un deber de los Estados (2018, p.20). Bien señala Benente (2019) que conceptualizar a la educación superior como derecho humano no solamente se debe ver como derecho individual que cada persona posee, sino que debe conceptualizarse como un derecho del pueblo a 
la educación superior, con el fin de concebir una educación no mercantil para que el pueblo haga universidad y la universidad se haga para el pueblo (p.105).

El ejercicio de este derecho permite el desarrollo de la personalidad en libertad y otorga las cualidades académicas y técnicas necesarias para el mejoramiento de la calidad de vida y se vuelve un vehículo para el desarrollo del reconocimiento de la identidad colectiva (Bolívar, 2010, p.193). En palabras de Unzué (2019), "la expansión de la educación superior, y dentro de ella de la universitaria ha resultado tradicionalmente un indicador relevante de desarrollo social” (p. 203).

La educación, al ser un derecho trascendental en la vida del ser humano, se enmarca dentro de varios principios como la igualdad, equidad, progresividad, eficiencia, responsabilidad, participación, calidad, entre otros; en los cuales el Estado, por medio de sus instituciones, deberá actuar para el desarrollo y aplicación de estos principios. La educación a lo largo de la vida del ser humano ha sido considerada más que un derecho, en sí es una de las claves del siglo XXI según la Declaración de Hamburgo sobre la Educación de Adultos², ya que la misma permite una ciudadanía activa y promueve la democracia, justicia y la igualdad entre hombres y mujeres; la educación da significado a la vida, y la misma debe ser garantizada por el Estado, dentro de sus deberes primordiales (1997).

La Convención Americana sobre Derechos Humanos en su artículo 26 referente al desarrollo de los DESC establece el compromiso de los Estados partes a adoptar actos normativos especialmente en educación, ciencia y cultura, en concordancia con el Protocolo de San Salvador ${ }^{3}$ que, en su artículo 13, establece el derecho a la educación a toda persona. Los tratados internacionales de derechos humanos

2 Declaración realizada en la Quinta Conferencia Internacional sobre Educación de Adultos del 14 al 18 de julio de 1997. Hamburgo, Alemania

3 Protocolo Adicional a la Convención Americana sobre Derechos Humanos en materia de derechos económicos, sociales y culturales "Protocolo de San Salvador". Ratificado por el Estado ecuatoriano el 25 de marzo de 1993. 
ratificados por el Estado ecuatoriano lo comprometen a garantizar, respetar y tutelar el ejercicio del derecho a la educación por los mecanismos: normativos y por medio de garantías jurisdiccionales, caso contrario por la imposibilidad de brindar recursos efectivos, accesibles y eficaces podría dar lugar a la búsqueda de justicia y, por ende, reparación integral en el Sistema Interamericano de Derechos Humanos, acorde al artículo 19, numeral 6, del Protocolo de San Salvador. Para que el Estado garantice el derecho a la educación debe asegurar que este derecho cumpla con ciertas características fundamentales, que se abordan en el siguiente enunciado.

\section{Características del derecho a la educación}

El derecho a la educación se encuentra dentro de los DESC, los cuales fueron declarados en el PIDESC; este derecho ha sido considerado como núcleo duro de los DESC, al ser un derecho que posibilita una vida digna y el goce de otros derechos fundamentales de las personas sintetizando la indivisibilidad e interdependencia de los derechos humanos (Pinto, 2004, p.72). La educación en todo nivel educativo, tiene cuatro características básicas, según lo previsto en la Observación General 13 del Comité de Derechos Económicos, Sociales y Culturales (1999): y estos son: a) disponibilidad; b) accesibilidad; c) aceptabilidad; y d) adaptabilidad.

La disponibilidad es, conforme lo contempla el Comité DESC en su Observación General No. 13 referente a la existencia de instituciones (de educación superior) y programas de enseñanza en cantidad suficiente dentro del Estado, una cantidad apropiada de centros educativos que permitirá a las personas mayores opciones para decidir a que institución ingresarán, y, sobre todo, permitir que una población porcentualmente alta acceda a la educación; sin embargo, la disponibilidad no acaba con la cantidad necesaria de instituciones dentro de una circunscripción territorial, sino que deben estas instituciones encontrarse dotadas de todas las condiciones necesarias para poder desenvolver un espacio correcto para transmitir conocimiento como es: edificios en un estado adecuado, instalaciones sanitarias, agua 
potable, docentes calificados con salarios competitivos, bibliotecas, y hoy podríamos añadir centros de informática y computación.

En relación a la accesibilidad plantea el Comité DESC que deben ser accesibles a todos sin discriminación, y esta accesibilidad contempla tres dimensiones: i) no discriminación; ii) material; y iii) económica (Comité DESC, 1999 E/C.12/1999/10).

En relación a la no discriminación, este acceso debe ser para todos, sin distinción alguna y generando un énfasis en los grupos en situación de vulnerabilidad. Este derecho debe verse materializado mediante las políticas que manejen las instituciones de educación superior en donde deben privilegiar un sistema de méritos para el acceso de los estudiantes, sin discriminación de sexo, etnia, idioma, discapacidad, factores económicos, culturales, sociales o religiosos (Sinchi y Gómez, 2018).

La Corte Constitucional prohibió la discriminación indirecta, misma que consiste en el resultado de la interpretación de normas, de su aplicación o de políticas públicas que supuestamente implementan dichas normas, más aún cuando los agentes que las interpretan ejecutan servicios públicos como la educación, deberán aplicar las normas sin generar discriminación (CC, 2020, Sentencia No. 189410-JP/20, párr.55).

Desde la dimensión material de la accesibilidad, la misma debe ser asequible, ya sea por su localización geográfica o a través del uso de herramientas tecnológicas. Esto significa que debe la educación ser alcanzada y no restringida su acceso. El avance en el uso de la tecnología, en especial las referidas a la información y comunicación digital, es un aspecto de vital importancia en el mundo actual, y el impacto de estos desarrollos en la educación es y será fundamental; sin embargo, los sistemas educativos latinoamericanos se enfrentan a un contexto con obstáculos financieros y tecnológicos que impiden equipararse adecuadamente, adicional a las complicaciones sociales que imposibilitan a cubrir los costos de conexión, velocidad que se transmiten los datos, dispositivos electrónicos y canales de comunicación virtuales (Villanueva, 2010, p.99). Por lo que, el uso de la 
tecnología en la educación superior pública sigue siendo una responsabilidad estatal y pendiente de trabajar arduamente.

La tercera dimensión de la accesibilidad se conceptualiza desde el enfoque económico, pues concibe a la educación como un derecho de alcance universal, implica la educación primaria propenderá a la gratuidad, y adicional, con relación a la educación superior establece el instrumento internacional que deberán los Estados implementar la gratuidad en estos niveles educativos. Este aspecto se encuentra relacionado directamente con el mandato constitucional que establece en el artículo 28, último párrafo, "[1]a educación pública será universal y laica en todos sus niveles, y gratuita hasta el tercer nivel de educación superior inclusive" (CRE, 2008).

Una de las medidas implementadas para mejorar la equidad en el acceso, entendiéndola como un bien público, ha sido la gratuidad de la educación ya que se eliminan los costos de matrícula y colegiatura (Rivera, 2019, p.3). La lógica de la política de gratuidad se relaciona con una visión social fundamentada en la igualdad de oportunidades, equidad en el acceso, e inclusión hacia grupos en situación de atención prioritaria, a través de la eliminación de las barreras de entrada en los costos de la matrícula (Rivera, 2019, p.9).

Al considerar a la aceptabilidad considera tanto en la forma y el fondo de la educación debe ser aceptable por los estudiantes, y cuando proceda, por los padres. El Estado como garante de este derecho deberá implementar estándares mínimos por medio de la normativa y políticas públicas para trazar los objetivos de la educación en todos los niveles.

Y la última característica que contempla el Comité DESC referente a la educación es la adaptabilidad, entendida como la flexibilización que tendrá este derecho, con la finalidad de adaptarse a las necesidades sociales, culturales de la población; acoplarse a los contextos culturales y/o sociales (Comité DESC, 1999 E/C.12/1999/10).

Las características descritas con anterioridad fueron recogidas en la sentencia 1894-10-JP/20 de la Corte Constitucional del Ecuador, en la 
cual se alude a la Observación General 13 del Comité DESC y se explica que dichas condiciones son aplicables en la educación pública como privada, cualquiera fuera la institución que permita obtener un título profesional o un grado académico a cualquier persona sin distinción.

De otro tanto, el Comité de Derechos Económicos, Sociales y Culturales (1999) afirma que la educación es un derecho humano y es el medio indispensable para realizar otros derechos, debido a que otorga los instrumentos necesarios a la persona para su autonomía, con la finalidad que haga plausible su proyecto de vida, evite la marginación económica y social de las personas y se genere una sociedad más justa y democratizada (Comité DESC, 1999 E/C.12/1999/10). En lo que respecta al ámbito de la educación superior, el derecho a la educación se caracteriza por ofrecer enseñanzas técnicas o profesionales para alcanzar un desarrollo cultural, social y económico que permita a los individuos de la sociedad ocupar un papel activo en la producción (Comité DESC, 1999 E/C.12/1999/10).

La Organización Internacional del Trabajo (OIT) establece, en el Convenio No. 142 (1975), que el Estado es el encargado de implementar un sistema educativo que permita el desarrollo técnico o profesional de las personas para que éstas integren plazas de empleo y dispongan de conocimiento que permitan el desarrollo personal, formen parte activa en los diferentes sectores económicos, sociales y culturales de la sociedad (OIT, C142). La importancia del rol del Estado en la educación consiste en generar políticas públicas que posibiliten las condiciones de acceso y permanencia en las instituciones educativas, ya que de esta manera las personas podrán obtener enseñanzas y conocimientos en igualdad de condiciones, sin discriminación alguna y la posibilidad de desarrollar actitudes que permitan su crecimiento personal y aporten a las necesidades de la sociedad (OIT, 1975, C141).

La educación, en definitiva, debe orientarse al desarrollo del sentido de la dignidad de la personalidad humana, debe capacitar a todas las personas para participar efectivamente en una sociedad libre y debe favorecer la comprensión entre todos los grupos étnicos, y entre las naciones y los grupos raciales y religiosos (Comité DESC, 1999 E/C.12/1999/10 núm. 4). 


\section{Relación entre el derecho a la educación y la vida digna}

Tanto la OIT (1975) como el Comité de DESC (1999) se refieren al rol que juega la educación en la realización personal del ser humano. Al respecto, las enseñanzas que adquiera la persona en cualquier institución educativa posibilitan que este desarrolle un proyecto de vida y satisfaga sus necesidades personales, económicas y sociales.

La Corte Constitucional Ecuatoriana prevé que el proyecto de vida consiste en el conjunto de expectativas razonables y accesibles a las posibilidades de la persona, empero su afectación debe evaluarse en caso de daños o pérdidas irreparables a las oportunidades del individuo; en definitiva, el proyecto de vida está ligado con la realización personal en base a las opciones que la persona pueda escoger y dirigir a un fin subjetivo (CC, Sentencia No. 1032-14-EP/19, 2019, párr.51).

El derecho a la vida digna está estrechamente relacionado con el derecho a la libertad, ya que, dependiendo de las oportunidades personales, la persona podrá decidir por sí misma las expectativas razonables y accesibles trazadas en el proyecto de vida (CC, Sentencia No. 140-18-SEP-CC, 2018). Este derecho mantiene una relación con la educación, al ser un derecho interdependiente y que al ser garantizado por el Estado permite a las personas elegir, dirigir y realizar su proyecto de vida con total autonomía, para así poder desarrollar sus habilidades y conocer sobre el mundo que le rodea.

La vida digna no debe conceptualizarse como la idea de vivir bien sin educación, lo propio será que la persona pueda ejercer este derecho en conjunto con una educación de calidad y accesible, así su proyecto de vida podrá ser encaminado al desarrollo. Este derecho se ejercerá en cuanto el Estado cumpla su rol preponderante en dotar a las universidades de presupuesto para que cumplan sus fines de enseñanza, vinculación con la sociedad y desarrollo del país, y esto se logrará con una garantía fundamental como es la autonomía universitaria. 


\section{Autonomía universitaria}

La educación superior podrá ser ejercida por medio de entidades las cuales contarán con aprobación estatal para prestar el servicio de educación (CRE, 2008, art.354), pero estas instituciones educativas tendrán un margen de actuación y, sobre todo, un respaldo normativo en lo relacionado a sus actuaciones: lo descrito se concibe como autonomía universitaria.

En virtud de lo anterior, el Estado es el ente encargado de garantizar la igualdad de condiciones de acceso a las universidades públicas, sin discrimen alguno, pero también debe dotar de autonomía a las instituciones universitarias para que determinen los parámetros objetivos para el acceso de los estudiantes, tales como pruebas de admisión, apertura de cupos dependiendo de las vacantes disponibles o la oferta restringida de cupos en carreras para mantener el prestigio profesional (Kisilevsky, 2002).

Esta posibilidad de actuar por parte de las instituciones de educación superior (en adelante IES) se verá plasmada en la autonomía de sus actividades, en el marco de lo estipulado en la Constitución que contempla el reconocimiento de la autonomía académica, administrativa, financiera y orgánica (CRE, 2008, art.355).

El Comité de DESC, en la Observación General No. 13 señala que la autonomía de las instituciones de educación superior es necesaria para adoptar las decisiones inherentes a su ámbito académico, a su gestión, normativa y a todas las actividades conexas (CC, Sentencia 140-18-SEP-CC, 2018). Es considerada una protección a las IES frente a posibles injerencias arbitrarias, por lo que se encontrará respaldada de manera normativa (Pacheco \& Pacheco, 2015) y hoy en día, se puede considerar desde un resguardo jurisprudencial por los criterios desarrollados por la Corte Constitucional del Ecuador en el panorama de la pandemia a causa del COVID-19. 


\section{Acontecimientos ocurridos en el Estado ecuatoriano en la pandemia COVID-19}

El presidente del Ecuador mediante Decreto Ejecutivo No. 1017, emitido el 16 de marzo de 2020, declaró el estado de excepción por calamidad pública en todo el territorio ecuatoriano por las consecuencias de la pandemia conocida como COVID-194. Esta declaración suspendió el ejercicio de los derechos de libertad, tránsito, asociación y reunión; y planteó, actuaciones estatales extraordinarias para detener la propagación tales como la movilización de las Fuerzas Armadas y organismos de la Administración Pública.

En lo referente al estado de excepción, esta es una situación extraordinaria que contempla el propio Estado constitucional con el fin de afrontar una circunstancia que desborda de la normalidad, situación que supera las alternativas de implementación y mecanismos de intervención que el ordenamiento jurídico prevé de manera ordinaria (CC, Dictamen No. 1-19-EE/19, 2019, párr.7), por lo que esta herramienta tiene un carácter temporal y excepcional y puede hacer uso el presidente de la República tal como determina la Constitución (2008, art.146) y dicha declaratoria tendrá que cumplir con características materiales y formales que la propia carta fundamental lo estipula.

La Corte Constitucional del Ecuador mediante Dictamen de Constitucionalidad 1-20-EE/20, expedido el 19 de marzo de 2020, realizó el correspondiente control de constitucionalidad y emitió dictamen favorable a la declaratoria de estado de excepción puntualizando en los considerandos 2 y 3 de la parte resolutiva, que las autoridades y personas que se encuentren ejerciendo potestades públicas deberán enmarcar sus competencias y atribuciones en lo que se encuentre expresamente establecido en la Constitución y la ley, así garantizando el principio de legalidad establecido en el artículo 226 de la Consti-

4 COVID-19 fue declarado oficialmente por la Organización Mundial de la Salud como pandemia el 11 de marzo de 2020. Más información: https://www.who.int/ $\mathrm{dg} /$ speeches/detail/who-director-general-s-opening-remarks-at-the-media-briefing-on-covid-19---11-march-2020. 
tución. Adicional, el órgano de justicia constitucional determinó que se deberá seguir lo que se encuentra estipulado en los artículos $164^{5}$ y 165 de la norma constitucional (CC, Dictamen 1-20-EE/20, 2020).

\section{En relación al artículo 165 de la Constitución se establece que:}

Durante el estado de excepción la Presidenta o Presidente de la República únicamente podrá suspender o limitar el ejercicio del derecho a la inviolabilidad de domicilio, inviolabilidad de correspondencia, libertad de tránsito, libertad de asociación y reunión, y libertad de información, en los términos que señala la Constitución.

Declarado el estado de excepción, la Presidenta o Presidente de la República podrá:

\section{(...)}

2. Utilizar los fondos públicos destinados a otros fines, excepto los correspondientes a salud y educación (CRE, 2008).

Haciendo hincapié frente a las circunstancias extraordinarias, imprevisibles y sobrevinientes de una calamidad pública, en la cual se decretó y contó con dictamen favorable de constitucionalidad que expresamente plantea el órgano constitucional que no podrá destinarse los fondos públicos de salud y educación para poder repeler o

$5 \quad$ Art. 164. La Presidenta o Presidente de la República podrá decretar el estado de excepción en todo el territorio nacional o en parte de él en caso de agresión, conflicto armado internacional o interno, grave conmoción interna, calamidad pública o desastre natural. La declaración del estado de excepción no interrumpirá las actividades de las funciones del Estado.

El estado de excepción observará los principios de necesidad, proporcionalidad, legalidad, temporalidad, territorialidad y razonabilidad. El decreto que establezca el estado de excepción contendrá la determinación de la causal y su motivación, ámbito territorial de aplicación, el periodo de duración, las medidas que deberán aplicarse, los derechos que podrán suspenderse o limitarse y las notificaciones que correspondan de acuerdo a la Constitución y a los tratados internacionales. 
actuar durante la calamidad pública. Esta garantía normativa establece que el estado no podrá eludir sus responsabilidades estatales en lo referente a la educación, aún en situaciones excepcionales.

El estado de excepción decretado por calamidad pública ${ }^{6}$ a causa del COVID-19, contemplaba una situación extraordinaria por causa de un virus desconocido, de fácil contagio que causa enfermedades respiratorias y muerte a personas en todo el mundo.

Frente a estos acontecimientos, el Estado ecuatoriano tomó decisiones económicas y financieras, entre las cuales la más relevante para la temática del presente artículo es el Oficio circular No. MEFVGF-2020-003-C (en adelante Oficio $003 \mathrm{MEF}$ ) expedido por el Viceministro de Economía y Finanzas en abril de 2020, mismo que estipuló un recorte a las instituciones de educación superior.

Frente a este oficio emitido por el Viceministro de Economía y Finanzas se presentaron en relación a la educación superior dos acciones ante la Corte Constitucional: i) acción de incumplimiento y ii) acción de inconstitucionalidad.

6 Entendida la calamidad pública en palabras de la Corte Constitucional del Ecuador como toda situación de catástrofe con origen en causas naturales o antrópicas que, por tener el carácter de imprevisible o sobreviniente, provoca graves consecuencias sobre la sociedad, particularmente, la lesión o puesta en riesgo de la integridad de la vida humana o de la naturaleza. La misma contará con dos elementos esenciales que deberán concurrir: i) la presencia de una situación catastrófica derivada de causas naturales o humanas que afecte gravemente a las condiciones sociales de una región o de todo el país; y ii) que la concurrencia de dicha situación sea imprevista o sobreviniente (Dictamen No. 1-20-EE/20 párr. 28 y 29). 


\section{i. Acción de incumplimiento}

En relación a la acción de incumplimiento, garantía establecida en el artículo 436 numeral $9^{7}$ con sustento en el artículo 86 numeral 3 y $4^{8}$ de la Constitución del Ecuador; que tiene por objeto el cumplimiento de sentencias y dictámenes constitucionales por las autoridades o particulares obligados a acatar lo determinado en las resoluciones jurisdiccionales constitucionales (Uribe, 2013, p.59) y procede cuando el fallo no se ha ejecutado integralmente, pues la misma carece de eficacia y por ende no garantiza una tutela judicial efectiva (Ávila, 2015, p.12).

Existieron cinco demandas de acción de incumplimiento ${ }^{9}$ en contra de la circular emitida por el Viceministro de Economía y Finanzas alegando un incumplimiento del Dictamen 1-20-EE/20 que declaraba la constitucionalidad del Decreto Ejecutivo que estableció el primer estado de excepción por causa del COVID-19. Las acciones fueron acumuladas por el Pleno de la Corte Constitucional ${ }^{10}$. Se

7 Art. 436.- La Corte Constitucional ejercerá, además de las que le confiera la ley, las siguientes atribuciones:

9. Conocer y sancionar el incumplimiento de las sentencias y dictámenes constitucionales.

8 Art. 86.- Las garantías jurisdiccionales se regirán, en general, por las siguientes disposiciones:

3. (...) Las sentencias de primera instancia podrán ser apeladas ante la corte provincial. Los procesos judiciales sólo finalizarán con la ejecución integral de la sentencia o resolución.

4. Si la sentencia o resolución no se cumple por parte de servidoras o servidores públicos, la jueza o juez ordenará su destitución del cargo o empleo, sin perjuicio de la responsabilidad civil o penal a que haya lugar. Cuando sea un particular quien incumpla la sentencia o resolución, se hará efectiva la responsabilidad determinada en la ley.

9 Asignadas con los números de causa: 34-20-IS, 35-20-IS, 36-20-IS, 38-20-IS y 3920-IS. Causas acumuladas aprobadas por el Pleno de la Corte Constitucional en las sesiones extraordinarias del 12 y 22 de mayo de 2020.

10 Decisión de 12 de mayo de 2020 en sesión extraordinaria del Pleno de la Corte Constitucional. 
presentó por varios accionantes la petición de medidas cautelares conjuntas y la Corte dispuso a la cartera de estado suspender y abstenerse de realizar modificaciones presupuestarias a las instituciones de educación superior y detener los efectos del oficio circular No. MEF-VFG-2020003-C hasta adoptar una decisión de fondo.

En las alegaciones de los accionantes se manifestaba incumplimiento de los numerales 2 y 3 de la parte resolutiva del dictamen constitucional, pues la actuación del órgano estatal afectaría a la accesibilidad y calidad de la educación superior, y se pretendía por medio de esta garantía jurisdiccional ordenar el incumplimiento del dictamen y evitar el recorte presupuestario y garantías de no repetición, con la finalidad que el Estado no vuelva a buscar recortar los recursos en el ámbito de educación.

Los accionados, instituciones estatales manifestaron, en audiencia pública de 28 de mayo de 2020, que no existía un recorte al presupuesto de la educación superior; sino más bien fue una reoptimización de recursos y búsqueda de eficiencia, alegando que no se incumplió el Dictamen 1-20-EE/20 y que la vía constitucional propuesta no era la idónea; en conclusión lo adoptado se encontraba dentro del margen de lo legal, y parte esta decisión de un proceso técnico de revisión periódica del presupuesto y de sus componentes, por lo que no existe como tal una reducción al presupuesto de las universidades (CC, Sentencia 34-20-IS y ac, 2020, párr. 50-65).

La Corte Constitucional al decidir sobre el fondo de las acciones de incumplimiento que alegaban inobservancia del dictamen en relación al punto 2 de la parte resolutiva del Dictamen 1-20-EE/20 que establecía que las actuaciones dentro del marco de un estado de excepción deberán encontrarse dentro de las competencias legalmente previstas. La Corte analizó la competencia del Viceministro de Economía y Finanzas para expedir las Directrices presupuestarias para el segundo trimestre del ejercicio fiscal 2020, acto que ejecutó por medio del oficio circular No. MEF-VFG-2020-003-C, y la Corte consideró que la competencia para adoptar las políticas económica, fiscal y tributaria acorde el artículo 261 numeral 5 de la Constitución (2008) son del Estado Central, misma que la ejerce el Ministerio de 
Economía y Finanzas y sus actuaciones se regulan en la normativa infralegal vigente que otorga la facultad de emitir actos resolutivos, por lo que tiene competencia para expedir esta circular (CC, Sentencia No. 34-20-IS y ac, 2020, párr. 100-110).

Otro punto fundamental del fallo referente a las acciones de incumplimiento en relación al presupuesto a las instituciones de educación superior, fue alegado por los accionantes el incumplimiento del punto 3 de la parte resolutiva del dictamen en donde estableció la Corte que "con sustento en los artículos 164 y 165 de la Constitución, se enfatiza que la suspensión de derechos y la adopción de medidas excepcionales, sólo puede ordenarse mediante decreto ejecutivo de Estado de excepción (...)" (CC, Dictamen No. 1-20-EE/20, 2020, pár.3), en suma a este punto establece el órgano constitucional que es atribución del Ministerio de Economía y Finanzas aumentar y/o rebajar los ingresos y gastos que modifiquen los niveles presupuestarios fijados y que el expedir el oficio circular no constituye una medida excepcional, sino una medida del régimen constitucional y legal ordinario, ajustándose a los criterios de pertinencia y oportunidad (CC, Sentencia No. 34-20-IS y ac, 2020, párr. 120 y 121).

Establece que no se evidencia disposición de los recursos de la educación superior a otros fines, y por ende no es factible, ordenar mediante sentencia que se restituya una cantidad de dinero que muy probablemente no se recaude en un ejercicio fiscal determinado, así desestimó las acciones de incumplimiento y revocó las medidas cautelares (CC, Sentencia 34-20-IS y ac, 2020, párr. 148 y 151).

Precedente jurisprudencial que determinó que la vía mediante acción de incumplimiento no era la adecuada, entendida por la Corte Interamericana de Derechos Humanos el recurso adecuado como el "recurso idóneo para proteger la situación jurídica infringida. En todos los ordenamientos internos existen múltiples recursos, pero no todos son aplicables en todas las circunstancias" (CorteIDH, Serie C No.9, 1989, párr.64) y que no se demostró por parte de los accionantes en sus alegaciones un incumplimiento de lo dispuesto por la Corte Constitucional en su dictamen de constitucionalidad del estado de excepción. 


\section{ii. Acción de inconstitucionalidad 9-20-IA/20}

Frente a la circular emitida por el Viceministro de Economía y Finanzas en abril de 2020 (considerada en discusión en la garantía jurisdiccional referida en el acápite anterior), se presentó ante la Corte Constitucional una acción de inconstitucionalidad, acción adicional que se planteó en contra del Oficio No. MEF-SP-2020-0002 emitido por la Subsecretaría del presupuesto del Ministerio de Economía y Finanzas y la Resolución RPC-SO-012- No.238-2020, expedida por el Consejo de Educación Superior en mayo de 2020.

La acción de inconstitucionalidad plantea una observancia de los requisitos constitucionales para la formación y emisión de las disposiciones jurídicas (CC, Sentencia No. 9-20-IA/20, 2020, párr.67) y sobre el fondo permite garantizar la adecuación de las normas que componen el ordenamiento jurídico al contenido de la Constitución (Guerrero, 2012, p.109), pues toda norma que fuere dictada en oposición a la misma, ya sea formal o material carecerá de validez jurídica y deberá ser declarada contraria a la Constitución y expulsarla del ordenamiento jurídico (Guerrero, 2012, p.103).

En concordancia con lo señalado la Corte Constitucional establece:

[...] la acción de inconstitucionalidad constituye el mecanismo de control abstracto a posteriori por excelencia. El control abstracto de constitucionalidad no es otra cosa que la posibilidad de examinar la constitucionalidad de una norma sin hacer referencia a un caso concreto, por lo que el control de constitucionalidad, previsto en el artículo 436 numeral 2 de la Constitución, se realiza con abstracción respecto de la aplicación concreta de la norma y se limita únicamente a determinar la conformidad o no del texto normativo impugnado con las disposiciones consagradas en la Constitución de la República. Si el resultado de este examen determina la inconstitucionalidad, el acto normativo impugnado será declarado inválido, garantizando con ello la supremacía y la fuerza normativa de la Constitución (CC, Sentencia No. 001-17-STC-CC, 2017). 
Al analizar la causa 9-20-IA/20, los accionantes alegaban que los actos impugnados desconocían el carácter normativo de la Constitución y su supremacía, violentando obligaciones internacionales en materia de derechos humanos, en particular con el derecho a la educación y otros derechos conexos al mismo; y frente al reconocimiento constitucional de la autonomía universitaria, en su administración, gestión financiera y económica y académica, esta se ve perjudicada por las actuaciones del Ministerio de Economía y Finanzas (MEF) y el Consejo de Educación Superior (CES).

Los órganos estatales entre sus posturas señalaron la competencia para expedir los actos normativos y mediante estas actuaciones el MEF, generó una reestimación de los presupuestos de todas las entidades que conforman el Presupuesto General del Estado como consecuencia de los ingresos disminuidos a causa de la pandemia COVID-19 y otros factores como caída del precio del petróleo; y, frente a esta reestimación no significa una afectación a la autonomía universitaria y a la calidad de la educación en general, ni la educación superior en particular.

La Corte Constitucional en el presente caso, al analizar la constitucionalidad por la forma de la circular del MEF contempla que tiene competencias constitucionales y legales en base al Código Orgánico de Planificación y Finanzas Públicas (COPLAFIP) que crea el Sistema Nacional de Finanzas Públicas (SINFIP), el cual le permite la rectoría y creación de normas políticas, y actuaciones que tengan por objeto gestionar los ingresos, gastos y financiamiento público ${ }^{11}$,

11 Código Orgánico de Planificación y Finanzas Públicas. Art. 70.- Sistema Nacional de Finanzas Públicas (SINFIP). El SINFIP comprende el conjunto de normas, políticas, instrumentos, procesos, actividades, registros y operaciones que las entidades y organismos del Sector Público, deben realizar con el objeto de gestionar en forma programada los ingresos, gastos y financiamiento públicos, con sujeción al Plan Nacional de Desarrollo y a las políticas públicas establecidas en esta Ley.

Todas las entidades, instituciones y organismos comprendidos en los artículos 225, 297 y 315 de la Constitución de la República se sujetarán al SINFIP, en los términos previstos en este código, sin perjuicio de la facultad de gestión autóno- 
considerando que dentro del sector público se encuentran las universidades, por lo que en concordancia con la Sentencia No. 34-20-IS y acumulados establece la competencia del órgano estatal para emitir este tipo de actuaciones.

Ahora bien, como se señaló previamente, la Corte Constitucional realizó un análisis de fondo para observar que la presente actuación se deriva y se fundamenta en otras superiores, ya que toda norma jurídica o acto administrativo con efectos generales debe encontrarse en concordancia con la Constitución de la República. En este precedente histórico la Corte aborda la inconstitucionalidad del Oficio circular MEF 003 expedido por el Viceministro de Economía y Finanzas en abril de 2020 y otros actos jurídicos de efectos generales, y se refiere a la autonomía universitaria desde el campo académico, administrativo y financiero; así como contempla como una verdadera garantía para el cumplimiento de los fines de las IES (CC, Sentencia No. 9-20-IA/20, 2020, párr.102).

En relación a la autonomía académica contempla la Corte como una condición fundamental e insoslayable para el cumplimiento de los fines relacionados con la investigación, búsqueda de la verdad, y producción de ciencia, tecnología, cultura y arte (CC, Sentencia No. 9-20-IA/20, 2020, párr.91), la misma que tendrá una protección constitucional, y directamente hace relación a que las instituciones de educación superior no podrán cumplir sus fines académicos en el caso de no contar con facultades para: organizar, gestionar, y administrar sus recursos (CC, Sentencia No. 9-20-IA/20, párr.92).

La autonomía administrativa y orgánica abarca la facultad de administrarse y organizarse, sin injerencias, contando con una libertad para que las IES definan la forma en que se organizan internamente

ma de orden administrativo, económico, financiero, presupuestario y organizativo que la Constitución o las leyes establecen para determinadas entidades.

Art. 71.- Rectoría del SINFIP.- La rectoría del SINFIP corresponde a la Presidenta o Presidente de la República, quien la ejercerá a través del Ministerio a cargo de las finanzas públicas, que será el ente rector del SINFIP. 
(Díaz, 2018, p.28), lo cual incluye el cogobierno universitario, permitiendo el protagonismo y responsabilidad en la toma de decisiones de todos los sujetos que conforman la comunidad universitaria: estudiantes, docentes, personal administrativo y autoridades para una legitimidad en las actuaciones y participación activa de actores estratégicos (Jara y Cedeño, 2018, p.7).

Con respecto a la autonomía financiera, la Corte Constitucional es enfática en que las IES tendrán la facultad de disponer de sus recursos, la optimización del gasto, la ejecución presupuestaria y la fiscalización, estos tópicos deberán encontrarse relacionados con el carácter solidario y responsable que tiene la autonomía universitaria, si bien no serán privados de sus rentas o asignaciones presupuestarias, ni mucho menos un retardo en los pagos por parte del Estado, es prioritario el manejo de estos valores de una manera eficaz y oportuna para así garantizar los principios relacionados a la educación superior y sus instituciones (CC, Sentencia No. 9-20-IA/20, 2020, párr. 95).

El análisis de la circular MEF 003 por la Corte Constitucional hace alusión a que este acto no fue un mero lineamiento o recomendaciones, sino más bien fueron restricciones efectivas a las IES, pues incluía de modo general una serie de limitaciones, condicionamientos y prohibiciones vinculadas con los egresos del personal y otros egresos presupuestarios de las IES públicas (CC, Sentencia No. 9-20IA/20, 2020, párr.144,152).

Si bien el Ministerio de Economía y Finanzas, en base al COPLAFIP, tiene la facultad como ente rector de las finanzas públicas de variar hasta un $15 \%$ para disminuir o aumentar los ingresos del sector público respecto a las cifras presupuestarias aprobadas por la Asamblea Nacional; en estas circunstancias se encontraba directamente relacionado las modificaciones a las preasignaciones, por lo que estas reformas en el presupuesto de las IES alteran su planificación institucional, la continuidad y ejecución de ciertas carreras, programas de investigación, pago de servicios y adquisición de bienes, imposibilitando la contratación de personal docente y administrativo requerido para su actividad normal y planificación institucional (CC, Sentencia No. 9-20-IA/20, 2020, párr. 148, 150, 151), lo 
cual generaría una afectación palpable a la autonomía universitaria y más aún incumpliendo el mandato constitucional que establece como prioridad la inversión en educación, frente a otras áreas de la inversión pública (CRE, 2008, art.355), criterio acertado por la Corte Constitucional para respetar el derecho a la educación superior.

El financiamiento que realiza el Estado a las instituciones de educación superior (IES) será un componente básico y necesario para una educación superior de calidad, sin embargo, no será el único, pues bien lo afirma Rodríguez (2007, p.72) que se deberá mantener una gestión seria, corresponsable, honesta y autocrítica para no terminar en un descalabro financiero que pague la sociedad, y dentro de ella los más pobres, por lo que las IES tendrán la posibilidad de acceder a un financiamiento y entrega del mismo en un tiempo oportuno, pero está deberá contener una responsabilidad y saber manejar los fondos de una manera eficiente para poder cumplir el principio de solidaridad y responsabilidad establecidos en la Constitución del Ecuador (CRE, 2008, art.355).

Esta garantía a las IES como es la autonomía universitaria debe ser respetada en las dimensiones que aborda la Corte Constitucional, pues así se garantizará una educación gratuita de calidad y supone la eliminación de las barreras de acceso a las instituciones universitarias, lo cual promueve la equidad socioeconómica y permite a las personas a generar y potenciar habilidades individuales que permiten satisfacer otras necesidades como la alimentación, vivienda, empleo, salud y alcanzar la superación personal, así como disfrutar de vacaciones, seguridad social, mejoramiento social y, en general, alcanzar su proyecto de vida (Ponce y Carrasco, 2016).

Si bien el Ecuador no excluye la educación privada, éste propugna que toda persona acceda a cualquier institución de educación superior de manera gratuita, lo cual contribuye al desarrollo del conocimiento, preservación y enriquecimiento de la cultura nacional y los saberes ancestrales, conforme los fines establecidos en la Ley Orgánica de Educación Superior (art. 8, letra c). 
La autonomía universitaria comprende la libertad de cátedra, designar sus autoridades, contratar docentes e investigadores, gestionar su ámbito administrativo, planificar y ejecutar su ejercicio económico y administrar sus recursos patrimoniales (CC, Sentencia No. 140-18-SEP-CC, 2018). En lo que respecta a las IES públicas, la reducción de presupuesto incide directamente en su autonomía financiera e impide que las instituciones educativas promuevan una labor académica de alta calidad que favorezca el desarrollo de la personalidad en libertad, fortalezca la producción investigativa y permita a la sociedad obtener las herramientas profesionales o técnicas necesarias para alcanzar una vida digna. El recorte presupuestario limita la autonomía de las IES públicas y fiscomisionadas, puesto que la falta de recursos no permitirá el correcto desarrollo de la labor académica y devendrá en un detrimento hacia la formación académica de los estudiantes.

La Corte Constitucional declaró inconstitucional por el fondo de la circular 003 del Ministerio de Economía y Finanzas, en relación con las medidas aplicables para las IES (CC, Sentencia No. 9-20-IA/20).

En este punto, la resolución RPC-SO-012-No-238-2020 emitida por el Consejo de Educación Superior, órgano encargado de la planificación, regulación y coordinación del sistema de educación superior (CRE, 2008, art.353), órgano que entre sus atribuciones se encuentra dictar la normativa reglamentaria para el ejercicio de sus competencias (LOES, 2012, art.166-169); dicha resolución se expide en el marco de la "Normativa transitoria para el desarrollo de actividades académicas en las Instituciones de Educación Superior, debido al estado de excepción decretado por la emergencia sanitaria ocasionada por la pandemia de COVID-19", la cual reformaba el Reglamento de Carrera y Escalafón del profesor de educación superior, norma que regula lo relacionado a: horas de docencia, planificación, vinculación del personal de apoyo, carrera y escalafón del personal académico de las IES (CES, RPC-SO-037-NO.265-2012).

El CES expidió su resolución con fundamento en la pandemia causada por el COVID-19 en el Ecuador y plantea cambios significativos al tiempo de dedicación a las horas de docencia, así como la contratación ocasional de docentes. En relación a las horas de docencia la disposición 
analizada en el presente caso aumentaba las horas de docencia, por lo que, solo se tomarán en cuenta como horas laborales las que el docente tenga contacto con los estudiantes (de forma presencial o virtual, sincrónica o asincrónica) tal como dispone el artículo 17 de la LOES, y no considerando las horas de aprendizaje autónomo entendido como "el conjunto de actividades de aprendizaje individuales o grupales desarrolladas de forma independiente por el estudiante sin contacto con el personal académico o el personal de apoyo académico" (LOES, 2010).

Por dicho anteriormente, la resolución del CES establecía un incremento hasta del $467 \%$ de horas de clase semanales, por ejemplo, en la dedicación del docente de tiempo completo, empero según el Reglamento de Carrera y Escalafón mínimo debían ser 3 horas y máximo 16. Con la Resolución del CES se modifica la carga horaria al considerar mínimo 14 horas y máximo 26; valores diferentes, pero no alejados ocurre con los docentes de medio tiempo y tiempo parcial. Lo cual a criterio de la Corte Constitucional de manera acertada significa una disminución desproporcionada del tiempo que un docente puede dedicar a la preparación de clase, a organizar y desarrollar otras actividades de docencia. Adicional, imposibilitando el realizar actividades de investigación, estudio de campo y vinculación con la comunidad (CC, Sentencia No. 9-20-IA/20, párr.172 y 173).

Esta resolución referente a la modificación de las horas de docencia, disminuye exponencialmente el tiempo de preparación de clase, en ciertos casos aproximadamente a treinta minutos para preparar cada hora de clase, lo que sería contrario a las garantías de mejoramiento pedagógico y académico del personal docente y generaría una deficiencia en la calidad y la propia Corte afirma que atentaría al principio de la educación superior, adicionalmente perjudica otros aspectos como el equilibrio de la docencia en generar otras actividades fundamentales dentro del campo universitario (CC, Sentencia No. 9-20-IA/20, 2020, párr. 178 y 179).

La Corte Constitucional en su fallo determina inconstitucionales tanto la circular 003 del Ministerio de Economía y Finanzas como la resolución del Consejo de Educación Superior donde reformaba el Reglamento de Escalafón y plantea a estos dos organismos que enmarquen 
sus actuaciones referente a racionalizar el gasto, optimización fiscal o reprogramación presupuestaria que se encuentre de por medio las instituciones de educación superior a la autonomía universitaria, la calidad de la educación superior y las particularidades de las IES; adicional que trabajen de manera coordinada con los diversos actores del sistema de educación superior: cuerpo docente, administrativo y estudiantes; y frente a estas decisiones económicas no deben anular las garantías de mejoramiento pedagógico y académico del personal de las IES. (CC, Sentencia No. 9-20-IA/20, 2020, pág. 57).

Para concluir este apartado se analiza a breves rasgos el voto salvado de los jueces constitucionales que se encontraron en contra del voto de mayoría. En relación a la circular 003 del MEF establecen que "no se encuentra una relación entre el referido oficio circular y el recorte presupuestario de las universidades denunciado por los accionantes" (Voto salvado Sentencia No. 9-20-IA/20, párr.6); ahora bien la prohibición o limitación de generar ciertas actuaciones establecidas en la circular 003 del MEF para el sector público (no desligando a las IES públicas) como señalaba la imposibilidad de contratación de personal docente y administrativo, este requerido para su actividad cotidiana de las IES, lo cual podría incurrir en suspensión o cierre de carreras, programas; adicional de pago de servicios generales, implementación de un sistema tecnológico para poder cubrir varios aspectos educativos en la pandemia a causa del COVID-19 no es un asunto aislado al presupuesto necesario para las IES pondría en grave riesgo la actividad de universidades y escuelas politécnicas de educación superior públicas, en su funcionamiento, mantenimiento y adecuación a una nueva normalidad.

Adicional establece el voto salvado que no puede desconocerse que a las IES les sea aplicable las directrices del oficio 003 del MEF y el efecto sería en los ámbitos detallados en este documento, que serían las cuestiones específicas relacionadas a temas netamente administrativos (Voto salvado Sentencia No. 9-20-IA/20, párr.7). Esta afirmación causa una preocupación, pues la autonomía universitaria no solo comprende la libertad de cátedra, designación de sus autoridades; adicionalmente mantiene un enfoque fundamental en la organización financiera como es la contratación de docentes e investigadores, 
gestión en su ámbito administrativo, planificación y ejecución de su ejercicio económico y administración de sus recursos patrimoniales (CC, Sentencia 140-18-SEP-CC, 2018). Por lo que la actividad administrativa de la universidad permite garantizar el cumplimiento de sus fines, la organización y manejo de su patrimonio y de brindar una atención de calidad y eficiente a estudiantes y personas que lo requieran, por lo que, el enfoque administrativo no está alejado a la autonomía universitaria.

A este aspecto de "afectación exclusiva al aspecto administrativo de las IES" referida en el voto salvado de la Corte Constitucional, la Comisión Interamericana de Derechos Humanos en el marco de los derechos humanos de las personas con COVID-19 expidió la Resolución No. 4/2020, que dispone a los Estados:

[...] prever dentro de los diferentes niveles de los sistemas educativos, la implementación de medidas que mitiguen la posible interrupción de los estudios y se enfoque en la reducción del abandono de los mismos. Así como atenuar otras consecuencias derivadas directamente de la pandemia, tomando especialmente en consideración el papel de la escuela en los entornos más vulnerables, como proveedora de higiene, salud o alimentos (CIDH, 27/07/2020, párr.42).

Esta disposición internacional no debe considerarse aislada del tratamiento y mejoramiento de la actividad administrativa de las IES, por lo que se encuentran en tiempos de pandemia es un gran reto de adaptar la actividad presencial a una virtual o híbrida, implementar mecanismos de atención oportuna por canales antes no usados o no muy frecuentes, y permitiendo así que la educación superior permanezca y siga siendo un espacio de aprendizaje y desarrollo de la sociedad.

En lo referente al análisis de la Resolución del Consejo de Educación Superior en el punto del incremento de las horas de trabajo al personal académico, el voto salvado plantea que es una decisión acertada, pues la misma se encuentra sustentada por la emergencia sanitaria que se encuentra el Ecuador a causa de la pandemia del COVID-19 y es una disposición transitoria, momentánea, mientras 
dure esta emergencia. En este punto la pandemia fue una causa imprevisible, que los gobiernos no pudieron prever ni imaginar la dimensión de la misma, por lo que, señalar una situación temporal, considerando que el Ecuador se mantuvo seis meses en estado de excepción por causa de esta pandemia, y que al momento de escribirse este texto se mantiene esta emergencia, ya no puede ser considerada transitoria, pues la misma según la OMS ha advertido a la población que la duración de esta pandemia podría prolongarse un largo tiempo (Cordero, 02/08/2020) y aumentar la carga horaria a los docentes podrá perjudicar a la educación sobre todo a la calidad, y a un trabajo precario y en contra del mejoramiento pedagógico y académico de sujetos que son protagonistas en la educación superior y enseñan a las nuevas generaciones para un desarrollo a la sociedad.

La Corte Interamericana de Derechos Humanos señaló que

[...] dada la naturaleza de la pandemia, los derechos económicos, sociales, culturales y ambientales deben ser garantizados sin discriminación a toda persona bajo la jurisdicción del Estado y, en especial, a aquellos grupos que son afectados de forma desproporcionada porque se encuentran en situación de mayor vulnerabilidad (Corte IDH, 9/04/2020, Declación 1/20).

La educación superior al ser parte de los DESC es de vital importancia tutelar el ejercicio de este derecho y más aún en tiempos complejos como es una pandemia, y es el derecho a la educación la posibilidad que tendrán las personas de acceder a un proceso de formación personal, social y cultural de carácter permanente que busque el acceso al conocimiento, a la ciencia, a la técnica y a los demás bienes y valores de la cultura, pues este derecho se configura como un bien de suma importancia para la sociedad (CCCol, Sentencia T646/11, 2011).

En medida de que el Estado tiene la obligación de desarrollar un sistema educativo que cumpla con las condiciones de disponibilidad, accesibilidad, adaptabilidad y aceptabilidad debe desarrollar políticas públicas que vayan de acuerdo a cumplir con estas obligaciones y no establecer medidas que su consecuencia sea la 
restricción al acceso a la educación superior por falta de entrega de recursos económicos por parte del Gobierno Nacional. Es imperativo recalcar que la educación es fundamental para el desarrollo de la ciudadanía y la libertad en sociedad.

\section{Conclusiones}

La educación es uno de los elementos prioritarios y que caracterizan el desarrollo de un país, por medio de este derecho se configura la posibilidad de ejercer otros tales como la vida digna. Las actividades estatales como son los actos normativos, políticas e inversiones públicas, deben perseguir un fin el cual permita que las personas puedan acceder, continuar y concluir su educación superior, y esto se ejecutará por medio de un constante y permanente manejo de todo el aparataje estatal, respetando la normativa constitucional y los instrumentos internacionales de Derechos Humanos que reconocen a la educación superior un derecho y una obligación del Estado ecuatoriano garantizar y permitir su ejercicio.

La existencia y establecimiento del derecho a la educación superior de manera textual en la Constitución, instrumentos internacionales de Derechos Humanos, y en más normas no será suficiente, pues el Estado por medio de sus actividades deberá garantizar mecanismos que permitan el ejercicio a la educación superior de las personas; pues en sí mismo este derecho permite el desarrollo de la sociedad, y no puede ser mercantilizado pues esto imposibilita y restringe su ejercicio, dejando de ser universal.

Es imperante señalar que la justiciabilidad del derecho a la educación dentro de los DESC, ha permitido una tutela de este derecho, y frente a violaciones del mismo permite su protección por parte de los órganos jurisdiccionales, por lo que, el derecho a la educación superior será plenamente exigible y tiene que garantizarse con el máximo de los recursos que dispone el Estado.

La Corte Constitucional del Ecuador se pronunció sobre la importancia del respeto al derecho a la educación superior desde la garantía 
a la autonomía universitaria de las instituciones de educación superior en el marco de las decisiones adoptadas por el Estado ecuatoriano en los primeros meses de pandemia por causa del COVID-19, y desarrolló un criterio fundamental de respeto a su presupuesto, al manejo de sus fondos y al trabajo de docentes y personal administrativo; pues se recalca que frente a medidas regresivas en la educación superior se tendrá un efecto multiplicador negativo incluso más allá de la educación misma, afectando las posibilidades de empleo, los planes de vida, el acceso a la cultura y la participación democrática. Estas limitaciones podrían condicionar la consecución de otros derechos.

\section{Referencias Bibliográficas}

Andrade Ortega, E. (2017). Universidad, Estado y Autonomía. En S. Cabrera, C. Cielo, K. Moreno y P. Ospina (eds.), Las reformas universitarias en Ecuador (2009-2016). Extravíos, ilusiones y realidades (pp. 53-69. Quito: Universidad Andina Simón Bolívar.

Ávila, F. (2015). Tesis "Acción de incumplimiento: fundamentos conceptuales y líneas jurisprudenciales de la Corte Constitucional del Ecuador para el periodo de transición". Programa de Maestría en Derecho Procesal. Universidad Andina Simón Bolívar. Sede Ecuador. Recuperado de: http:// repositorio.uasb.edu.ec/bitstream/10644/4664/1/T1722-MDP-Avila-Incumplimiento.pdf

Benente, M. El derecho humano a la educación superior. Una revisión a la luz de los 70 años de la gratuidad. "Donde antes estaba solamente admitido el oligarca". La gratuidad de la educación superior, a 70 años. Mauro Benente (compilador). EdunPaz, Editorial Universitaria.

Bolívar, L. (2010). El derecho a la educación. Revista Instituto Interamericano de Derechos Humanos Volumen 52 Recuperado de http://www.corteidh. or.cr/tablas/r25566.pdf 
Comité de América Latina y el Caribe para la Defensa de los Derechos de la Mujer - CLADEM. (2011). Jurisprudencia sobre el Derecho a la Educación en los Sistemas Internacionales de Protección de los Derechos Humanos. Lima, Perú.

Comboni, S. \& Júarez, J. (1997). La educación superior en América Latina: perspectivas frente al siglo XXI. Revista Política y Cultura, (9). Recuperado de https://www.redalyc.org/articulo.oa?id=267/26700902

Cóndor, V. (2018). Influencia de la Reforma de Córdova en la universidad ecuatoriana. Revista Conrado, 14(63), (pp. 15-19). Recuperado de http:// conrado.ucf.edu.cu/index.php/conrado

Cordero, Á. (02/08/2020). La OMS advierte que la pandemia durará un "largo tiempo". France 24. Recuperado de https://www.france24.com/ es/20200802-oms-tedros-pandemia-coronavirus-covid19

Cotino, L. (2012). El derecho a la educación como derecho fundamental. Especial atención a su dimensión social prestacional. Madrid: Centro de Estudios Políticos y Constitucionales.

Díaz, D. (2018). El cogobierno como elemento consustancial de la autonomía universitaria responsable en el marco jurídico ecuatoriano. Disertación previa a la obtención de abogado. Pontificia Universidad Católica del Ecuador. Recuperado de http://repositorio.puce.edu.ec/bitstream/handle/22000/15938/TESIS\%20EMPASTADO.pdf?sequence=1\&isAllowed=y

Ferreyra, M., Avitabile, C., Botero, J., Haimovich, F. y Urzúa, S. (2017). Resumen Momento decisivo. La educación superior en América Latina y el Caribe. Banco Mundial. Washington, DC., EE.UU. Recuperado de https://openknowledge.worldbank.org/bitstream/handle/10986/26489/211014ovSP. pdf? sequence $=5 \&$ isAllowed $=y$

Guerrero, J. (2012). Aproximación al control abstracto en Ecuador. La acción de inconstitucionalidad. Apuntes de Derecho Procesal Constitucional. Cuadernos de trabajo. Tomo 3. Corte Constitucional para el período de Transición. Centro de Estudios y Difusión del Derecho Constitucional. Quito, Ecuador. Juan Montaña Pinto edit. 
Jara, I. \& Cedeño, J. (2018). El cogobierno en la educación superior en Ecuador: práctica de la gobernanza. Revista Científica ECOCIENCIA. Universidad Ecotect. Volumen 5. No. 5. Recuperado de http://revistas.ecotec.edu.ec/ index.php/ecociencia/article/view/121/87

Kisilevsky, M. (2002). Condiciones sociales y pedagógicas de ingreso a la educación superior en la Argentina. Dos estudios sobre el acceso a la educación superior en la Argentina. Buenos Aires: IIPE-UNESCO.

León, A. (2007). ¿Qué es la educación? Educere. Universidad de los Andes. Volumen 11. No. 39. Venezuela. Recuperado de https://www.redalyc.org/ pdf/356/35603903.pdf

Navas, M. \& Chiliquinga, L. Acceso y gratuidad en la universidad desde un enfoque de derechos. Reflexiones sobre el caso ecuatoriano. "Donde antes estaba solamente admitido el oligarca”. La gratuidad de la educación superior, a 70 años. Mauro Benente (compilador). EdunPaz, Editorial Universitaria.

Pacheco, L. \& Pacheco, R. (2015). Evolución de la educación superior en el Ecuador. La Revolución Educativa de la Universidad Ecuatoriana". Pacarina del Sur. Año 6 No. 23. Recuperado de http://pacarinadelsur.com/home/amautas-y-horizontes/1128-evolucion-de-la-educacion-superior-en-el-ecuador-la-revolucion-educativa-de-la-universidad-ecuatoriana\#resumen

Ponce, J. y Carrasco, F. (2016). Acceso y equidad a la educación superior y posgrado en el Ecuador, un enfoque descriptivo. Revista Latinoamericana de Políticas y Acción Pública, 3(2), (pp. 9-22). ISSN 1390-9193.

Organización de Estados Iberoamericanos para la Educación, Ciencia y Cultura (OEI). (s.f). Breve evolución histórica del Sistema Educativo. Capítulo 2. Sistemas Educativos Nacionales - Ecuador.

Olivier, G. Retos de la educación superior privada en América Latina: entre la expansión y la resistencia. IdeAs. Recuperado de https://journals.openedition.org/ideas/382?lang=en

Pareja, F. (1986). La educación superior en Ecuador. Centro Regional para la Educación Superior en América Latina y el Caribe - CRESALC - UNES- 
CO. Caracas, Venezuela. Recuperado de https://unesdoc.unesco.org/ ark:/48223/pf0000072628

Pinto, M. (2004). Los derechos económicos, sociales y culturales y su protección en el sistema universal y en el sistema interamericano. Revista Instituto Interamericano de Derechos Humanos. No. 40. Recuperado de http://www. corteidh.or.cr/tablas/R08064-2.pdf

Rivera, J. (2019). A 10 años de la gratuidad en la educación superior: ¿qué pasó con el acceso? Revista Chakiñan, (7). Recuperado de http://scielo.senescyt.gob.ec/scielo.php?script=sci_arttext\&pi$\mathrm{d}=\mathrm{S} 2550-67222019000100058 \&$ lang $=$ es

Rivera, J. (2019). La gratuidad de la educación superior y sus efectos sobre el acceso: Caso Ecuador. Archivos Analíticos de Políticas Educativas, 27. Recuperado de https://www.uasb.edu.ec/documents/10181/2364056/Jairo+Rivera/ccab93e5-df81-48c8-8173-f417dd8537b4

Rodríguez, F. (2007). El financiamiento de la Educación Superior en América Latina: Una visión panorámica. Informe sobre la educación superior en América Latina y el Caribe 2000-2005. Instituto Internacional para la Educación Superior en América Latina y el Caribe. Organización de las Naciones Unidas para la Educación, la Ciencia y la Cultura.

Simon, F. (24 de noviembre de 2005). La educación en la Constitución Ecuatoriana de 1998. Derechoecuador.com Diario La Hora. Recuperado de https://www.derechoecuador.com/la-educacioacuten-en-la-constitucioacuten-ecuatoriana-de-1998

Sinchi, E. y Gómez, G. (2018). Acceso y deserción en las universidades. Alternativas de financiamiento. Alteridad, 13(2). Recuperado de http://scielo.senescyt.gob.ec/scielo.php?script=sci_arttext\&pi$\mathrm{d}=\mathrm{S} 1390-86422018000200274 \&$ lang $=\mathrm{es}$

Unzué, M. (2019). Una mirada sobre la educación superior en América Latina. Revista de Educación y Derecho No. 19. Universitat de Barcelona. Recuperado de https://revistes.ub.edu/index.php/RED/article/view/28357/29055 
Uribe, D. (2013). Cumplimiento de sentencias y dictámenes constitucionales. Apuntes de Derecho Procesal Constitucional Cuadernos de trabajo. Tomo 2. Corte Constitucional para el periodo de Transición. Centro de Estudios y Difusión del Derecho Constitucional. Quito, Ecuador. Juan Montaña Pinto y Angélica Porras Velasco edit.

Villanueva, E. (2010). Perspectivas de la educación superior en América Latina: construyendo futuros. Perfiles educativos Volumen No. 129. Universidad Nacional Autónoma de México, Instituto de Investigaciones sobre la Universidad y la Educación. Recuperado de http://www.scielo.org.mx/scielo. php?script=sci_arttext\&pid=S0185-26982010000300006

\section{Jurisprudencia constitucional}

Corte Constitucional del Ecuador. Sentencia No. 001-17-STC-CC. Caso No. 0039-07-TC de 11 de enero de 2017. Jueza ponente: Ruth Seni Pinoargote.

Corte Constitucional del Ecuador. Sentencia No. 140-18-SEP-CC. Caso No. 1764-17/EP de 18 de abril de 2018. Jueza ponente: Tatiana Ordeñana Sierra.

Corte Constitucional del Ecuador. Dictamen No. 1-19-EE/19. Casos No. 1-19EE y 2-19-EE acumulados de 30 de mayo de 2019. Jueza ponente: Carmen Corral Ponce.

Corte Constitucional del Ecuador. Sentencia No. 1032-14-EP/19. Caso No. 103214-EP de 18 de diciembre de 2019. Jueza ponente: Daniela Salazar Marín.

Corte Constitucional del Ecuador. Sentencia No. 1894-10-JP/20. Caso No. 189410-JP de 4 de marzo de 2020. Jueza ponente: Teresa Nuques Martínez.

Corte Constitucional del Ecuador. Dictamen No. 1-20-EE/20. Caso No. 1-20EE de 9 de marzo de 2020. Jueza ponente: Teresa Nuques Martínez.

Corte Constitucional del Ecuador. Sentencia No. 34-20-IS/20 y acumulados. Caso No. 34-20-IS/20 y acumulados de 31 de agosto de 2020. Jueza ponente: Carmen Corral Ponce. 
Corte Constitucional del Ecuador. Sentencia No. 9-20-IA/20. Caso No. 9-20-

IA de 31 de agosto de 2020. Juez ponente: Agustín Grijalva Jiménez.

\section{Jurisprudencia internacional}

Corte Interamericana de Derechos Humanos. Caso Velásquez Rodríguez vs. Honduras. Reparaciones y Costas. Sentencia de 21 de julio de 1989. Serie C No. 7.

\section{Jurisprudencia comparada}

Corte Constitucional Colombiana. Sentencia T646/11, de 1 de septiembre de 2011.

\section{Normas jurídicas}

Asamblea Constituyente Constitución de la República del Ecuador 20 de octubre de 2008. Registro Oficial 449.

Asamblea Nacional del Ecuador. Ley Orgánica de Educación Superior 12 de octubre de 2010. Registro Oficial Suplemento No. 298.

Asamblea Nacional del Ecuador. Código Orgánico de Planificación y Finanzas Públicas 22 de octubre de 2010. Registro Oficial Suplemento No. 306.

Consejo de Educación Superior. Reglamento de carrera y escalafón del profesor de educación superior 08 de noviembre de 2017.

\section{Instrumentos internacionales}

Asamblea General de la Organización de las Naciones Unidas en su resolución 2200 A. Pacto Internacional de Derechos Económicos, Sociales y Culturales. Ratificado por el Ecuador y publicado en el Registro Oficial no. 101 año I con fecha 24 de enero de 1969. Entrada en vigor: 3 de enero de 1976. 
Comisión Interamericana de Derechos Humanos Resolución No. 4/2020, Derechos Humanos de las personas con COVID-19 de 27 de julio de 2020.

Comité de Derechos Económicos, Sociales y Culturales. Organización de Naciones Unidas. Observación General No. 13. El derecho a la educación (artículo 13 del Pacto). 21 periodo de sesiones, 15 de noviembre al 3 de diciembre 1999.

Corte Interamericana de Derechos Humanos Declaración No. 1/20. COVID-19 y Derechos Humanos: los problemas y desafíos deben ser abordados con perspectiva de Derechos Humanos respetando las obligaciones internacionales de 9 de abril de 2020. San José, Costa Rica.

Instituto Internacional para la Educación Superior en América Latina y el Caribe de la Organización de las Naciones Unidas para la Educación, la Ciencia y la Cultura (UNESCO). Declaración de la III Conferencia Regional de Educación Superior para América Latina y el Caribe (CRES), celebrada entre el 11 y el 15 de junio de 2018. Córdoba, Argentina.

Organización de Estados Americanos. Convención Americana sobre Derechos Humanos 22 de noviembre 1969.

Organización de Estados Americanos. Protocolo Adicional a la Convención Americana sobre Derechos Humanos en materia de derechos económicos, sociales y culturales "Protocolo de San Salvador". Ratificado por el Estado ecuatoriano el 25 de marzo de 1993.

Organización de las Naciones Unidas para la Educación, la Ciencia y la Cultura (UNESCO). Declaración realizada en la Quinta Conferencia Internacional sobre Educación de Adultos del 14 al 18 de julio de 1997. Hamburgo, Alemania

Organización Internacional del Trabajo. Convenio No. 142: Convenio sobre el desarrollo de los recursos humanos. 1975. 